\title{
Social Health Literacy Assets
}

Janine Bröder, Orkan Okan, Torsten M. Bollweg, Dirk Bruland, Paulo Pinheiro, Ullrich Bauer

\section{Source}

Janine Bröder, Orkan Okan, Torsten M. Bollweg, Dirk Bruland, Paulo Pinheiro, Ullrich Bauer. (2019). Child and Youth Health Literacy: A Conceptual Analysis and Proposed Target-Group-Centred Definition. IJERPH, vol. 16 (18), 3417.

Namely, the social and cultural resources one can access via present social support structures in the close social environment (family/peer/community context). This also points to the importance of the health literacy available to individuals and groups within their social environment and that, as such, is also part of the children's health literacy. 